\title{
FORMAS DE FÓSFORO NO SOLO SOB PASTAGENS NATURAIS SUBMETIDAS À ADIÇÃO DE FOSFATOS ${ }^{(1)}$
}

\author{
Leandro Bittencourt de Oliveira ${ }^{(2)}$, Tales Tiecher ${ }^{(3)}$, Fernando Luiz Ferreira de Quadros ${ }^{(4)}$, \\ José Pedro Pereira Trindade ${ }^{(5)}$, Luciano Colpo Gatiboni ${ }^{(6)}$, Gustavo Brunetto ${ }^{(7)}$ \& Danilo \\ Rheinheimer dos Santos ${ }^{(8)}$
}

\begin{abstract}
RESUMO
A maioria das pastagens naturais no bioma Campos no sul do Brasil cresce em solos com baixa disponibilidade de fósforo $(\mathrm{P})$, mas com altos teores de $\mathrm{P}$ total e de $\mathbf{P}$ orgânico. Este trabalho objetivou avaliar as alterações nas formas de $\mathbf{P}$ no solo, ao longo de um ciclo de crescimento de pastagens naturais, decorrentes da aplicação de fontes de fosfato. Em três experimentos instalados em áreas de pastagem natural, foi aplicado $P$ nas formas de hiperfosfato de Gafsa, superfosfato triplo e testemunha, arranjados em blocos casualizados com três repetições. Nos experimentos instalados no município de Candiota, RS, em pastagens naturais sob Luvissolo Úmbrico e Neossolo Litólico, foram aplicados $100 \mathrm{~kg} \mathrm{ha}^{-1} \mathrm{de} \mathrm{P}_{2} \mathrm{O}_{5} \mathrm{em}$ setembro de 2010. Na pastagem sob Argissolo Vermelho no município de Santa Maria, RS, foram aplicados 180, 90, 100 e $100 \mathrm{~kg} \mathrm{ha}^{-1} \mathrm{de}_{2} \mathrm{O}_{5}$ nos anos de 1997, 1998, 2002 e 2010, respectivamente. Amostras de solo foram coletadas $(0-10 \mathrm{~cm})$ ao longo da estação de crescimento da pastagem (0, 55, 116, 171 e 232 dias, após aplicação do fosfato em Candiota; e 0, 50, 83, 129, 159 e 186 dias, após aplicação do fosfato em Santa Maria). Foram analisados os teores de $\mathbf{P}$ disponível por resina de troca aniônica, o $\mathrm{P}$ imobilizado na biomassa microbiana do solo, o $\mathrm{P}$ extraído por $\mathrm{NaOH}$ 0,1 mol $\mathrm{L}^{-1}$, o $\mathrm{P}$ orgânico total e o $\mathrm{P}$ total. Os teores de $\mathrm{P}$ disponível aumentaram rapidamente com a aplicação de fosfato solúvel, mas no final do período de avaliação esses se equivaleram ao do fosfato natural, que foi semelhante à testemunha. A
\end{abstract}

(1) Recebido para publicação em 17 de junho de 2013 e aprovado em 28 de janeiro de 2014.

(2) Doutorando em Zootecnia, Departamento de Zootecnica, Universidade Federal de Santa Maria - UFSM. Av. Roraima, 1000, Bairro Camobi. CEP 97105-900 Santa Maria (RS). Bolsista do CNPq. E-mail: leandroliveira86@hotmail.com

(3) Doutorando em Ciência do Solo, Departamento de Solos, UFSM. Bolsista da CAPES. E-mail: tales.t@hotmail.com

(4) Professor Associado, Departamento de Zootecnia, UFSM. Bolsista do CNPq. E-mail: flfquadros@yahoo.com.br

(5) Pesquisador, EMBRAPA Pecuária Sul. BR 153, km 603. CEP 96401-970 Bagé (RS). E-mail: Jose.Pereira-Trindade@embrapa.br

(6) Professor Associado, Departamento de Solos, Universidade do Estado de Santa Catarina. Bolsista do CNPq. Av. Luis de Camões, 2090, Bairro Conta Dinheiro. CEP 88520-000 Lages (SC). E-mail: gatiboni@cav.udesc.br

(7) Professor Adjunto, Departamento de Solos, UFSM. Bolsista CNPq. E-mail: brunetto.gustavo@gmail.com

(8) Professor Associado, Departamento de Solos, UFSM. Bolsista do CNPq. E-mail: danilonesaf@gmail.com 
aplicação de fertilizantes fosfatados sob pastagens naturais com baixa disponibilidade de $\mathbf{P}$ aumentou a importância das frações inorgânicas lábeis às plantas, tornando-as menos dependentes da mineralização das frações orgânicas. As frações orgânicas, inclusive o P microbiano, não são bons indicadores da biodisponibilidade de $\mathrm{P}$ em pastagens naturais sob Argissolos, Neossolos e Luvissolos do sul do Brasil fertilizadas com fosfatos.

Termos de indexação: fósforo microbiano, disponibilidade de fósforo, ecossistemas campestres, fosfato solúvel e natural.

\title{
SUMMARY: FORMS OF SOIL PHOSPHORUS IN NATURAL PASTURES SUBJECTED TO ADDITIONS OF PHOSPHATES
}

\begin{abstract}
Most natural pastures in the Campos biome in Southern Brazil grow in soils with low phosphorus $(P)$ availability, but with high contents of total and organic $P$. The present study aimed to evaluate the changes in forms of soil P resulting from the application of phosphate sources over a growth cycle of natural pastures. In three experiments under natural pasture, $P$ was applied in the form of Gafsa rock phosphate, triple superphosphate, and a control treatment in a randomized block design with three replications. In the trials carried out in natural pastures in the municipality of Candiota $(R S)$ in an Alfisol and an Entisol, $100 \mathrm{~kg} \mathrm{ha} \mathrm{A}^{-1}$ of $\mathrm{P}_{2} \mathrm{O}_{5}$ was applied in September 2010. In natural pasture in an Ultisol in the municipality of Santa Maria (RS), 180, 90, 100, and $100 \mathrm{~kg} \mathrm{ha}^{-1}$ of $\mathrm{P}_{2} \mathrm{O}_{5}$ were applied in the years 1997, 1998, 2002, and 2010, respectively. Soil samples were collected $(0-10 \mathrm{~cm})$ throughout the natural grassland growing season $(0,55,116,171$, and 232 days after $P$ application at Candiota and 0, 50, 83, 129, 159, and 186 days after application of phosphate at Santa Maria). The levels of available $P$ were analyzed by anion exchange resin, as well as $P$ immobilized in the soil microbial biomass, $P$ extracted by $0.1 \mathrm{~mol} L^{-1} \mathrm{NaOH}$, total organic $P$, and total $P$. The available $P$ content increased rapidly with the application of soluble phosphate, but at the end of the evaluation period it was equivalent to the Gafsa rock phosphate, which was similar to the control. The application of phosphate fertilizers on natural pastures with low Pavailability increased the importance of the labile inorganic P forms to the plants, making them less dependent on the mineralization of organic $P$ fractions. The organic fractions, including microbial $P$, are not good indicators of the bioavailability of $P$ in natural pastures under Alfisols, Entisols, and Ultisols in Southern Brazil fertilized with phosphates.
\end{abstract}

Index terms: microbial phosphorus, phosphorus availability, grassland ecosystems, rock and soluble phosphate.

\section{INTRODUÇÃO}

Os solos sob ecossistemas de pastagens naturais no Rio Grande do Sul (RS) são ácidos e apresentam baixa disponibilidade de fósforo (P). Mesmo assim, a vegetação natural, na estação de crescimento, alcança taxas de acúmulo de matéria de $10,3 \mathrm{~kg} \mathrm{ha}^{-1} \mathrm{dia}^{-1}$ (Soares et al., 2005) até $26,9 \mathrm{~kg} \mathrm{ha}^{-1} \mathrm{dia}^{-1}$ (Pellegrini et al., 2010). A variação deve-se a fatores como tipo de solo, clima regional, em especial a disponibilidade hídrica e composição botânica da pastagem. Altas produções de forragem são possíveis porque em ambientes naturais equilibrados a biociclagem do $\mathrm{P}$ orgânico do solo, do $\mathrm{P}$ contido nos tecidos vegetais e na biomassa microbiana do solo (BMS) atende às necessidades das plantas (Oliveira et al., 2011). Todavia, há possibilidades de aumentar a produtividade das áreas de pastagens naturais, evitando a sua substituição por culturas anuais, florestas ou pastagens cultivadas (Tiecher et al., 2013). Nesse sentido, a aplicação de fertilizantes fosfatados sobre as pastagens tem evidenciado-se rentável economicamente e com impactos ambientais desprezíveis (Gatiboni et al., 2003).

Os fertilizantes fosfatados solúveis normalmente possuem elevado custo. Por isso, os fosfatos naturais (FN) podem ser uma alternativa economicamente mais viável para os pecuaristas. No entanto, a disponibilização de P pela aplicação de FN é sensível às elevações nos teores de $\mathrm{Ca}$, aos aumentos no $\mathrm{pH}$ e à queda do teor de umidade do solo, além da pequena área de contato solo/fertilizante quando é aplicado sobre as pastagens, desfavorecendo-o na comparação aos fosfatos solúveis. Entretanto, em solos ácidos $(\mathrm{pH}<5,2)$ e com baixos teores de $\mathrm{Ca}$, a eficiência agronômica dos FN em longo prazo pode ser suficientemente elevada para obterem-se rendimentos compatíveis à aplicação dos fosfatos solúveis (Gatiboni et al., 2003; Chien et al., 2011), apresentando-se adequados para o uso em culturas perenes (Szilas et al., 2007). Nesse sentido, Tiecher et al. (2013) demonstraram que após 154 meses o teor de $\mathrm{P}$ 
disponível no solo e a produção anual de forragem foram equivalentes com a aplicação de hiperfosfato de Gafsa e fosfatos solúveis. Contudo, o aumento de produção de forragem no inverno, período de baixo crescimento das pastagens naturais no sul do Brasil, só foi possível com a introdução de espécies exóticas de inverno e aplicação de fertilizantes fosfatados solúveis, que promoveram aumento rápido de $\mathrm{P}$ disponível no solo, fornecendo assim as condições necessárias para o estabelecimento do trevo vesiculoso (Trifolium vesiculosum) e do azevém (Lolium multiflorum).

O censo comum de que grande parte dos fosfatos solúveis aplicados nos solos subtropicais é "fixado" indefinidamente aos coloides tem sido amplamente contestado pelos trabalhos do grupo de pesquisa liderado por Rheinheimer, tanto em solos arenosos com baixos teores de sesquióxidos quanto em solos argilosos ricos em sesquióxidos (Rheinheimer et al., 2000; Rheinheimer \& Anghinoni, 2001; Rheinheimer et al., 2003a,b; Tiecher et al., 2012a). Além da rápida e quase total reversibilidade da adsorção de $\mathrm{P}$, a elevação do teor de $\mathrm{P}$ disponível no solo promove o aumento do teor de P imobilizado na BMS (Rheinheimer et al., 2008), que é um reservatório dinâmico de $P$ potencialmente disponível às plantas que se ajusta conforme o fornecimento de energia, de $\mathrm{C}$ e de $\mathrm{P}$ ao sistema (Oliveira et al., 2011). A imobilização de P no tecido da BMS diminui e retarda a adsorção do fosfato nos grupos funcionais de superfície de partículas inorgânicas do solo (Conte et al., 2002); posteriormente, com a sua morte e lise das células, o $\mathrm{P}$ pode ser mineralizado mais sincronizadamente com a demanda de crescimento das plantas, servindo como uma reserva de $\mathrm{P}$ disponível no curto e no médio prazo (Martinazzo et al., 2007). Além disso, em razão do aumento da produção de biomassa vegetal e da BMS poderá haver ainda maior conversão do $\mathrm{P}$ inorgânico aplicado via fertilizantes em formas menos lábeis de P orgânico, evitando a adsorção de fosfato solúvel pelo solo e servindo como uma reserva de $\mathrm{P}$ disponível no médio e no longo prazo (Tiecher et al., 2012b).

Os sítios de troca do solo capazes de adsorver P são finitos e possuem diferente afinidade pelo íon fosfato. Por isso, as frequentes aplicações de fosfato na superfície do solo sem revolvimento criam uma região de saturação dos sítios mais reativos, onde grande parte do $\mathrm{P}$ solúvel que não é absorvido pelas plantas ou imobilizado pela BMS é adsorvida com menor energia pelo solo, podendo tornar-se facilmente disponível (Rheinheimer \& Anghinoni, 2001; Conte et al., 2003; Kuo et al., 2005; Gatiboni et al., 2007, 2008; Takeda et al., 2009; Vu et al., 2010; Wang et al., 2010). Além disso, a matéria orgânica presente nos solos sob pastagens naturais, normalmente em teores elevados, pode auxiliar na rápida saturação dos grupos funcionais porque esses estão poucos disponíveis para a troca de ligantes em razão da interação prévia e natural dos sítios de adsorção com ácidos orgânicos de baixo peso molecular, que exercem competição com os íons fosfatos (Jones, 1998). Dessa forma, a adição de fosfatos, principalmente os solúveis, cria um ambiente fértil propício ao melhor desenvolvimento das espécies nativas e das exóticas introduzidas nas pastagens naturais do sul do Brasil, diminuindo a dependência do $\mathrm{P}$ disponibilizado pela ciclagem biológica e aumentando a produção de forragem.

Este trabalho objetivou avaliar o efeito da aplicação de fontes de fosfato sobre as formas de P no solo ao longo de um ciclo de crescimento de pastagens naturais em três experimentos conduzidos em diferentes solos do Rio Grande do Sul, com e sem histórico de aplicação de $P$.

\section{MATERIAL E MÉTODOS}

\section{Descrição das áreas de estudo e tratamentos}

Este trabalho foi constituído de três experimentos conduzidos em diferentes regiões fisiográficas do RS. Dois experimentos (I e II), com histórico recente de aplicação de $\mathrm{P}$, foram instalados em 28 de setembro de 2010 numa propriedade de pecuária com base em pastagem natural no município de Candiota. Esse está situado na zona de transição entre a região fisiográfica da serra do Sudeste e a região da Campanha sulriograndense $\left(31^{\circ} 25^{\prime} \mathrm{S}, 53^{\circ} 34^{\prime} \mathrm{O}\right)$, sendo o I, sob um solo Luvissolo Úmbrico; e o II, sob um Neossolo Litólico. O clima da região, segundo a classificação de Köppen, é do tipo subtropical Cfa, mesotérmico, com precipitação pluvial média anual de $1.350 \mathrm{~mm}$, temperatura média anual de $18{ }^{\circ} \mathrm{C}$, ocorrência de geadas de abril a novembro e estiagens frequentes de novembro a maio. A temperatura e precipitação pluvial média mensal histórica e para o período de estudo estão apresentadas na figura 1 . As áreas experimentais foram cercadas e a vegetação roçada a $5 \mathrm{~cm}$ da superfície do solo, sendo os resíduos retirados da área experimental. Em seguida, os tratamentos arranjados em blocos ao acaso com três repetições foram implantados e consistiram de uma testemunha sem aplicação de $\mathrm{P}$ e aplicação superficial de $100 \mathrm{~kg}_{\text {ha }}{ }^{-1}$ de $\mathrm{P}_{2} \mathrm{O}_{5}$, na forma de superfosfato triplo (SFT) e fosfato natural (hiperfosfato de Gafsa). Para calcular a quantidade de $\mathrm{P}$ aplicada, considerou-se o teor total de $\mathrm{P}_{2} \mathrm{O}_{5}$ do hiperfosfato de Gafsa (28\%) e o teor de $\mathrm{P}_{2} \mathrm{O}_{5}$ solúvel em citrato neutro de amônio + água do SFT (46\%). Todas as parcelas $\left(6,25 \mathrm{~m}^{2}\right.$ cada) receberam $30 \mathrm{~kg} \mathrm{ha}^{-1}$ de $\mathrm{N}$ na forma de ureia; não foi adicionado fertilizante potássico em razão de o teor de $\mathrm{K}$ no solo dos dois experimentos ter sido classificado como muito alto (CQFSRS/SC, 2004) (Quadro 1).

$\mathrm{O}$ terceiro experimento (III), com um histórico de 13 anos de adubação fosfatada, foi instalado em uma pastagem natural na área experimental do Departamento de Solos da Universidade Federal de Santa Maria (UFSM), situada na região fisiográfica da Depressão Central do RS (29 $49^{\circ}$ ' S, $53^{\circ} 42^{\prime}$ O), sob 
um Argissolo Vermelho. O clima da região é classificado como subtropical úmido $\mathrm{Cfa}$, segundo classificação de Köppen, com precipitação pluvial média anual de $1.769 \mathrm{~mm}$, temperatura média anual de

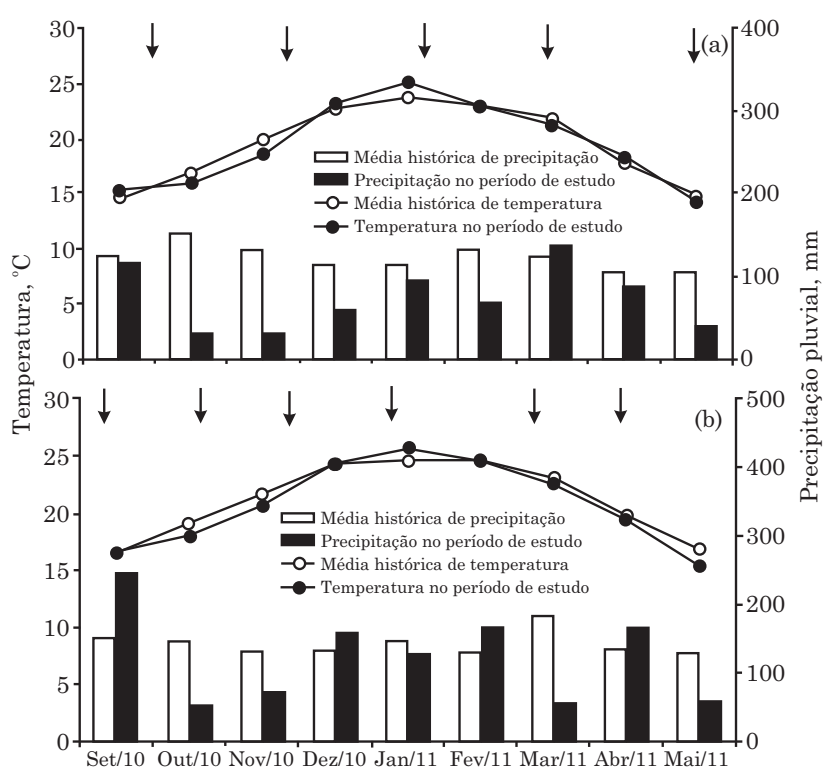

Figura 1. Temperatura média e precipitação média nas áreas experimentais de (a) Candiota (experimentos I e II) e (b) Santa Maria (experimento III). Primeira flecha indica data de aplicação das fontes de fosfato (início do experimento) e as demais indicam as datas das avaliações da massa de forragem. $19,2^{\circ} \mathrm{C}$ e ocorrência de geadas nos meses de maio a agosto. A temperatura e precipitação pluvial média mensal histórica para o período de estudo são apresentadas na figura 1 . O experimento foi instalado em 1997, em blocos ao acaso com três repetições, e os tratamentos consistiram da aplicação superficial de $180 \mathrm{~kg} \mathrm{ha}^{-1} \mathrm{de}_{2} \mathrm{O}_{5}$ nas formas de hiperfosfato de Gafsa e SFT e de uma testemunha, sem aplicação de P. Para calcular a quantidade de $\mathrm{P}$ aplicada, consideraram-se o teor total de $\mathrm{P}_{2} \mathrm{O}_{5}$ do hiperfosfato de Gafsa $(28 \%)$ e o de $\mathrm{P}_{2} \mathrm{O}_{5}$ solúvel em citrato neutro de amônio + água do SFT (46\%). Em toda a área experimental, foram aplicados $130 \mathrm{~kg} \mathrm{ha}^{-1}$ de $\mathrm{K}_{2} \mathrm{O}$, na forma de cloreto de potássio. Em 1998 e 2002, foram reaplicados 90 e $100 \mathrm{~kg} \mathrm{ha}^{-1}$ de $\mathrm{P}_{2} \mathrm{O}_{5}$, respectivamente. Nos invernos de 1997, 1998 e 2002, foram introduzidas na pastagem natural as espécies azevém (Lolium multiflorum) e trevo vesiculoso (Trifolium vesiculosum) por meio de semeadura direta depois de roçadas. Desde então, a pastagem ficou sob crescimento livre. Em 27 de agosto de 2010, a pastagem foi roçada a $5 \mathrm{~cm}$ da superfície do solo e, em seguida, foram retirados da área experimental os resíduos. Logo depois, foram aplicados $30 \mathrm{~kg} \mathrm{ha}{ }^{-1}$ de $\mathrm{N}$ na forma de ureia em todos os tratamentos e $100 \mathrm{~kg} \mathrm{ha}^{-1}$ de $\mathrm{P}_{2} \mathrm{O}_{5}$ na forma de hiperfosfato de Gafsa e SFT. Assim, as avaliações apresentadas neste trabalho foram obtidas na pastagem com o histórico de aplicação de $470 \mathrm{~kg} \mathrm{ha}^{-1}$ de $\mathrm{P}_{2} \mathrm{O}_{5}$ em cada fonte de $\mathrm{P}$.

\section{Coleta de solo e análise de $\mathbf{P}$}

Nos três experimentos, foi coletado solo na camada de 0-10 cm (oito subamostras) antes da aplicação dos

Quadro 1. Resumo das aplicações das fontes de fosfato e características físicas e químicas, na camada 0-10 $\mathrm{cm}$, nos solos antes da instalação dos experimentos

\begin{tabular}{|c|c|c|c|}
\hline Variável & $\begin{array}{l}\text { perimento I } \\
\text { solo Úmbrico }\end{array}$ & $\begin{array}{c}\text { Experimento II } \\
\text { Neossolo Litólico }\end{array}$ & $\begin{array}{c}\text { Experimento III } \\
\text { Argissolo Vermelho } \\
\end{array}$ \\
\hline \multicolumn{4}{|l|}{ Aplicação da fonte de fosfato } \\
\hline \multicolumn{4}{|l|}{ Meses após início do experimento em } \\
\hline Quantidade de $\mathrm{P}_{2} \mathrm{O}_{5}$ aplicada em cada aplicação $\left(\mathrm{kg} \mathrm{ha}^{-1}\right)$ & 100 & 100 & $180+90+100+100$ \\
\hline Quantidade total de $\mathrm{P}_{2} \mathrm{O}_{5}$ aplicada $\left(\mathrm{kg} \mathrm{ha}^{-1}\right)$ & 100 & 100 & 470 \\
\hline \multicolumn{4}{|l|}{ Atributo do solo } \\
\hline $\mathrm{pH}\left(\mathrm{H}_{2} \mathrm{O}\right)(1: 1 \mathrm{v} / \mathrm{v})$ & 4,6 & 4,9 & 4,5 \\
\hline Argila $\left(\mathrm{g} \mathrm{kg}^{-1}\right)$ & 190 & 190 & 170 \\
\hline Matéria orgânica $\left(\mathrm{g} \mathrm{dm}^{-3}\right)$ & 28 & 33 & 18 \\
\hline $\mathrm{P}^{(1)}\left(\mathrm{mg} \mathrm{dm}^{-3}\right)$ & 5,7 & 2,6 & 1,4 \\
\hline $\mathrm{K}^{(2)}\left(\mathrm{mg} \mathrm{dm} \mathrm{m}^{-3}\right)$ & 164 & 124 & 60 \\
\hline $\mathrm{Ca}^{2+}\left(\mathrm{cmol}_{\mathrm{c}} \mathrm{dm}^{-3}\right)$ & 2,7 & 3,4 & 1,2 \\
\hline $\mathrm{Mg}^{2+}\left(\mathrm{cmol}_{\mathrm{c}} \mathrm{dm}^{-3}\right)$ & 0,8 & 1,5 & 0,7 \\
\hline $\mathrm{Al}^{3+}\left(\mathrm{cmol}_{\mathrm{c}} \mathrm{dm}^{-3}\right)$ & 0,9 & 0,5 & 1,3 \\
\hline $\mathrm{CTC}_{\mathrm{pH} 7,0}\left(\mathrm{cmol}_{\mathrm{c}} \mathrm{dm}^{-3}\right)$ & 10,2 & 10,7 & 8,0 \\
\hline Saturação por bases (\%) & 39 & 49 & 40 \\
\hline Saturação por Al (\%) & 18 & 9 & 22 \\
\hline
\end{tabular}

(1) Extraído por RTA; ${ }^{(2)}$ extraído por Mehlich-1. 
tratamentos para a caracterização dos solos estudados. Nessas amostras de solo, foram avaliados teor de argila, matéria orgânica, $\mathrm{pH}\left(\mathrm{H}_{2} \mathrm{O}\right), \mathrm{P}$ e K disponíveis e $\mathrm{Al}$, Ca e Mg trocáveis (Tedesco et al., 1995) (Quadro 1).

Nos experimentos de Candiota, após a aplicação dos tratamentos, foram coletadas amostras de solo da camada de $0-10 \mathrm{~cm}$ (oito subamostras) nos dias $22 / 11 /$ $10,22 / 01 / 11,18 / 03 / 11$ e 18/05/11, correspondentes a $55,116,171$ e 232 dias após a aplicação dos tratamentos, respectivamente. No experimento de Santa Maria, as coletas foram realizadas nos dias 16/10/2010, 18/11/2010, 03/01/2011, 03/02/2011 e 01/03/2011, correspondendo a 50, 83, 129, 159 e 186 dias após a aplicação dos tratamentos, respectivamente. Nessas amostras de solo, foram avaliados o teor de $\mathrm{P}$ microbiano, $\mathrm{P}$ disponível por resina, $\mathrm{P}$ extraído por $\mathrm{NaOH} 0.1 \mathrm{~mol} \mathrm{~L}^{-1}$, P orgânico e P total. Após cada coleta, as amostras de solo foram acondicionadas em caixas térmicas com gelo. Logo depois, o solo foi peneirado em malha de $2 \mathrm{~mm}$ e armazenado em incubadora $\mathrm{a} \pm 4^{\circ} \mathrm{C}$, até a realização da análise de $\mathrm{P}$ imobilizado na BMS. Posteriormente, o solo foi seco em estufa com circulação forçada de ar a $\pm 65{ }^{\circ} \mathrm{C}$ e armazenado para as demais análises.

O P imobilizado na BMS foi estimado pelo método de fumigação-extração, após extração de P inorgânico com resina de troca aniônica (RTA) (Hedley \& Stewart, 1982a). Três conjuntos (A, B e C) de amostras de solo $(1,0 \mathrm{~g})$ foram agitados com $10 \mathrm{~mL}$ de água destilada e uma lâmina de RTA por $16 \mathrm{~h}$ a $25^{\circ} \mathrm{C}$. Posteriormente, as lâminas de RTA foram retiradas, o solo foi centrifugado por $15 \mathrm{~min}$ a $2.510 \mathrm{~g}$ e o sobrenadante foi descartado. Um conjunto de amostra de solo (A) foi fumigado com $1 \mathrm{~mL}$ de clorofórmio (livre de álcool) por $24 \mathrm{~h}$ a $25^{\circ} \mathrm{C}$, e os outros dois conjuntos (B e C) foram mantidos sob a mesma condição sem clorofórmio (não fumigado), adicionando $1 \mathrm{~mL}$ de água destilada para manter a proporção. A recuperação do $\mathrm{P}$ armazenado na BMS foi corrigida pela sorção de $\mathrm{P}$ inorgânico durante a extração, utilizando uma concentração de $50 \mathrm{mg} \mathrm{kg}^{-1}$ de solo no conjunto C. O P liberado nas amostras fumigadas e não fumigadas foi extraído com $10 \mathrm{~mL}$ de $\mathrm{NaHCO}_{3} 0,5 \mathrm{~mol} \mathrm{~L}^{-1}$ (ajustado a $\mathrm{pH} 8,5)$ por $16 \mathrm{~h}$ (relação solo:extrator 1:10) e centrifugado por $15 \mathrm{~min}$ a $2.510 \mathrm{~g}$. Posteriormente, os extratos dos três conjuntos de solo (A, B e C) foram digeridos com ácido sulfúrico $\left(\mathrm{H}_{2} \mathrm{SO}_{4}\right)$ e persulfato de amônia $\left(\left(\mathrm{NH}_{4}\right)_{2} \mathrm{~S}_{2} \mathrm{O}_{8}\right)$ em autoclave a $121^{\circ} \mathrm{C}$ (USEPA, 1971), com subsequente determinação de $P$, de acordo com método descrito por Murphy \& Riley (1962). Finalmente, o teor de P armazenado na BMS foi calculado pela equação proposta por Morel et al. (1996):

$$
\mathrm{P} \text { armazenado na BMS }=\frac{50\left([\mathrm{P}]_{\mathrm{A}}-[\mathrm{P}]_{\mathrm{B}}\right)}{0,4\left([\mathrm{P}]_{\mathrm{C}}-[\mathrm{P}]_{\mathrm{B}}\right)}
$$

em que 0,4 é o fator de recuperação $\left(K_{\mathrm{p}}\right)$ utilizado, assumindo que somente $40 \%$ do $\mathrm{P}$ armazenado na BMS foram extraídos pelo método (Brookes et al., 1982).
$\mathrm{O}$ teor de $\mathrm{P}$ disponível por resina de troca aniônica (RTA) foi determinado conforme segue: $1,0 \mathrm{~g}$ de solo foi acondicionado em tubos falcon de $15 \mathrm{~mL}$. Em seguida, foram adicionados $10 \mathrm{~mL}$ de água destilada e uma lâmina de RTA (placas AR 103 QDP 434 Ionics Inc.), saturada com $\mathrm{NaHCO}_{3} 0,5 \mathrm{~mol} \mathrm{~L}^{-1}$ a pH 8,5. Após, procedeu-se agitação por $16 \mathrm{~h}$, em agitador tipo ‘sem fim' a $25^{\circ} \mathrm{C}$. As lâminas foram então retiradas e lavadas com jatos de água destilada e depois eluídas em $10 \mathrm{~mL}$ de $\mathrm{HCl} 0,5 \mathrm{~mol} \mathrm{~L}^{-1}$. No extrato de $\mathrm{HCl}$, o teor de $\mathrm{P}$ foi determinado conforme descrito por Murphy \& Riley (1962).

O P moderadamente lábil foi estimado pela adição de10 mL de $\mathrm{NaOH} 0,1 \mathrm{~mol} \mathrm{~L}^{-1}$ em 1,0 g de solo em tubos falcon de $15 \mathrm{~mL}$. Posteriormente, as amostras foram agitadas por $16 \mathrm{~h}$ em agitador tipo 'sem fim' a $25^{\circ} \mathrm{C}$ com posterior centrifugação por $15 \mathrm{~min}$ a $2.510 \mathrm{~g}$. Os extratos foram digeridos com $\mathrm{H}_{2} \mathrm{SO}_{4}$ e persulfato de amônia em autoclave a $121{ }^{\circ} \mathrm{C}$ (USEPA, 1971). Finalmente, os extratos digeridos foram submetidos à determinação de $\mathrm{P}$ (Murphy \& Riley, 1962).

O P orgânico estimado pelo método de ignição foi obtido pela diferença entre a quantidade de $\mathrm{P}$ extraída com $\mathrm{H}_{2} \mathrm{SO}_{4} 0,5 \mathrm{~mol} \mathrm{~L}^{-1}$ de amostras de solo ignificadas $\left(550{ }^{\circ} \mathrm{C}, 2 \mathrm{~h}\right)$, bem como não ignificadas (Olsen \& Sommers, 1982). O P total do solo foi estimado pela digestão com $\mathrm{H}_{2} \mathrm{SO}_{4}$ e $\mathrm{H}_{2} \mathrm{O}_{2}$ na presença de $\mathrm{MgCl}_{2}$ saturado (Olsen \& Sommers, 1982). Todos os extratos foram reservados e submetidos à determinação de $\mathrm{P}$, conforme proposto por Murphy \& Riley (1962).

\section{Coleta de massa verde e análise de $\mathbf{P}$}

A massa de forragem acumulada foi avaliada no dia 18/05/11 (232 dias de crescimento) nos experimentos de Candiota e no dia 01/03/2011 (186 dias de crescimento) no experimento de Santa Maria, coincidindo com a data da última coleta de amostras de solo. Como antes da aplicação dos tratamentos todos os experimentos haviam sido roçados a $5 \mathrm{~cm}$ de altura, essa avaliação estimou a massa de forragem acumulada das pastagens durante o período de maiores temperaturas do ano, que é o período de maior crescimento das pastagens naturais no sul do Brasil. Essa avaliação foi realizada em quatro subamostras de $0,25 \mathrm{~m}^{2}$ por parcela. O material coletado foi seco em estufa com circulação forçada de ar a $\pm 65^{\circ} \mathrm{C}$ até peso constante e determinada a produção de matéria seca. A matéria seca vegetal foi submetida à digestão sulfúrica e análise do teor de $\mathrm{P}$, conforme Tedesco et al. (1995). Finalmente, foi realizado um balanço teórico de $\mathrm{P}$, considerando a variação do teor de $\mathrm{P}$ disponível por RTA no solo da camada $0-10 \mathrm{~cm}$ entre a primeira e a última coleta de solo e a quantidade de $\mathrm{P}$ acumulado na biomassa da parte aérea da pastagem natural.

\section{Análises estatísticas}

Os dados obtidos foram normalizados, transformando-os para log $(n+0,5)$, antes de realizar 
a análise da variância. Para a análise da variância (ANOVA) das formas de P no solo, o modelo estatístico adotado foi um bifatorial com parcelas subdivididas no tempo, em três blocos casualizados como repetições, assim descrito:

$$
\begin{aligned}
& Y_{i j k}=\mu+B_{i}+F_{j}+\operatorname{erro} a(i, j)+D_{k}+\operatorname{erro} b(i, k)+ \\
& F D_{j k}+\operatorname{erroc}(i, j, k)
\end{aligned}
$$

em que $\mu=$ média experimental; $B=$ blocos $(i=1,2$, 3); $F=$ fonte de $\mathrm{P}(j=1,2,3) ; D=$ dias após a aplicação das fontes de $\mathrm{P}(k=1,2,3,4$, para os experimentos de Candiota; e $k=1,2,3,4,5$, para o experimento de Santa Maria); e erro = erro experimental. Quando os efeitos dos tratamentos foram significativos a $\mathrm{p}<0,05$ pelo teste de $\mathrm{F}$, as diferenças entre as médias das fontes de fosfatos em cada época de coleta foram comparadas pelo teste de Tukey a p $<0,05$ e ajustadas equações de regressão para as épocas de amostragem.

\section{RESULTADOS}

A aplicação de fosfato solúvel na forma de superfosfato triplo (SFT) aumentou, em relação ao teor original do solo, 3,0; 4,2 e 18,3 vezes no teor de $\mathrm{P}$ disponível na primeira coleta dos experimentos I, II e III, respectivamente (Figura 2 a,b,c). Mesmo com o decréscimo natural com o passar do tempo, a disponibilidade manteve-se superior àquela da testemunha no final do período de avaliação (232 dias).

Nos experimentos I e II em Candiota, com aplicação recente de fosfato, o teor de $\mathrm{P}$ disponível diminuiu paulatinamente nos tratamentos testemunha e fosfato natural (FN) ao longo das avaliações realizadas (Figura 2a,b). A aplicação de FN resultou no aumento médio de apenas 1,4 vez no teor de $\mathrm{P}$ disponível, comparativamente à testemunha, mas diferiu dessa apenas na última coleta de solo. Já no experimento de Santa Maria, com histórico de 13 anos totalizando aplicação de $470 \mathrm{~kg} \mathrm{ha}^{-1}$ de $_{2} \mathrm{O}_{5}$, o teor de $\mathrm{P}$ disponível com a aplicação de $\mathrm{FN}$ foi em média (das quatro avaliações temporais) 4,3 vezes superior à testemunha, e nas últimas duas coletas foi equivalente ao SFT.

$\mathrm{O}$ teor de $\mathrm{P}$ microbiano não foi alterado pela aplicação de fontes de fosfato e pelas épocas de avaliação no experimento II, mas observou-se interação entre esses dois fatores nos experimentos I e III (Quadro 2). No experimento I, o teor de P microbiano aumentou com a aplicação de SFT apenas na terceira coleta (Figura 2d); e no III, o teor de P microbiano também aumentou com a aplicação de FN apenas na última coleta de solo, mas foi menor do que o teor obtido com a aplicação de SFT (Figura 2f).

Os teores de $\mathrm{P}$ extraído por $\mathrm{NaOH}$, $\mathrm{P}$ orgânico e $\mathrm{P}$ total não apresentaram interação entre as fontes de fosfato e as épocas de coleta nos três experimentos (Quadro 2). O teor de $\mathrm{P}$ extraído por $\mathrm{NaOH}$ não diferiu entre as épocas de avaliação em todos os tratamentos nos três experimentos (Quadro 2). Comparativamente à testemunha, nos experimentos com histórico recente de aplicação de $\mathrm{P}$ (Candiota), o teor de $\mathrm{P}$ extraído por $\mathrm{NaOH}$ com a aplicação de SFT aumentou 18 e $35 \%$ no Luvissolo e no Neossolo (experimentos I e II), respectivamente (Quadro 3). Já no experimento com histórico de 13 anos de aplicação de $\mathrm{P}$ (Santa Maria), o teor de $\mathrm{P}$ extraído por $\mathrm{NaOH}$ aumentou 42 e $88 \%$ nos tratamentos com a aplicação de FN e SFT, respectivamente (Quadro 3).

Nos experimentos I e II, com aplicação recente de $\mathrm{P}$, os teores de $\mathrm{P}$ total não foram alterados pela aplicação das fontes de fosfatos e não foram diferentes entre as épocas de coleta (Quadro 3). Da mesma forma, os teores de $\mathrm{P}$ orgânico não foram alterados pela aplicação das fontes de fosfatos, mas durante as coletas realizadas aos 114 e 170 dias de avaliação, que foi o período mais quente do ano (meses de janeiro e março); esses teores foram menores em todos os tratamentos (Figura 3). Já no experimento III, com o histórico de 13 anos de aplicação de $\mathrm{P}$, os teores de $\mathrm{P}$ total e de $\mathrm{P}$ orgânico não foram diferentes entre as épocas de coleta. Nesse experimento, o P orgânico aumentou em relação à testemunha aproximadamente 1,1 e 1,2 vezes com a aplicação de FN e SFT, respectivamente, e o P total aumentou aproximadamente 1,25 vez com a aplicação de FN e SFT (Quadro 3).

No experimento com histórico de 13 anos de aplicação de $\mathrm{P}$, o aumento no teor de $\mathrm{P}$ total do solo na forma orgânica foi de 17,4 \%, com a aplicação de FN $\left[\left(\mathrm{P}\right.\right.$ orgânico ${ }_{\mathrm{FN}}-\mathrm{P}$ orgânico testemunha $){ }^{*} 100 /\left(\mathrm{P}\right.$ total $_{\mathrm{FN}}$ $\mathrm{P}$ total $\left.\left._{\text {testemunha }}\right)\right]$, e de $22,4 \%$, com a aplicação de SFT $\left[\left(\right.\right.$ Porgânico $_{\mathrm{SFT}}$ - P orgânico testemunha $^{*}{ }^{*} 100 /\left(\mathrm{P}_{\text {total }} \mathrm{SFT}_{\mathrm{ST}}\right.$ $\mathrm{P}$ total testemunha $_{\text {) }}$.

No tratamento com aplicação de SFT, o acúmulo de $\mathrm{P}$ na parte aérea da massa de forragem foi responsável por apenas $29 \%$ da diminuição do teor de P disponível no solo da camada de $0-10 \mathrm{~cm}$ nos dois experimentos de Candiota, enquanto no experimento III, em Santa Maria, o acúmulo de $\mathrm{P}$ na parte aérea das plantas foi responsável por $52 \%$ da diminuição do teor de $\mathrm{P}$ disponível nessa mesma camada (Quadro 4). Isso significa que na última coleta de solo realizada no tratamento com aplicação de SFT aproximadamente 71 e $48 \%$ do $\mathrm{P}$ solúvel aplicado via SFT foram adsorvidos pelo solo, apresentando assim menor disponibilidade às plantas no curto prazo.

\section{DISCUSSÃO}

A aplicação de SFT foi mais eficiente em elevar o teor de $\mathrm{P}$ disponível do solo comparativamente ao $\mathrm{FN}$, como pode-se verificar pelos teores de P extraído por RTA nas primeiras coletas de solo dos três experimentos (Figura 2a,b,c). A aplicação de FN somente possibilitou disponibilidade de $\mathrm{P}$ equivalente ao fosfato solúvel na última avaliação, embora as doses 
tenham sidas as mesmas, em razão do decréscimo na disponibilidade de P do SFT que ocorreu em parte pela absorção de P pela pastagem, como observado por SornSrivichai et al. (1988) (29 e $52 \%$ para os experimentos I e II, em Candiota, e para o experimento III, em Santa Maria, respectivamente), e em parte pela adsorção de $\mathrm{P}$ pelo solo (Quadro 4), e não pelo aumento da disponibilidade proporcionada pelo FN. Isso demonstra que a disponibilização de $\mathrm{P}$ do $\mathrm{FN}$ no curto e no médio prazo é muito baixa, especialmente onde a concentração de Ca e o pH do solo são maiores, como no experimento II de Candiota (Dias, 1978). Para o período avaliado, a aplicação de FN não apresentou disponibilização de $\mathrm{P}$ ao longo do tempo nos experimentos de Candiota e apenas um pequeno incremento no teor de $\mathrm{P}$ disponível na segunda coleta no experimento III em Santa Maria, por causa do histórico de 13 anos de fertilização. Apesar disso, esse aumento foi inferior ao obtido com a aplicação de fosfato solúvel.
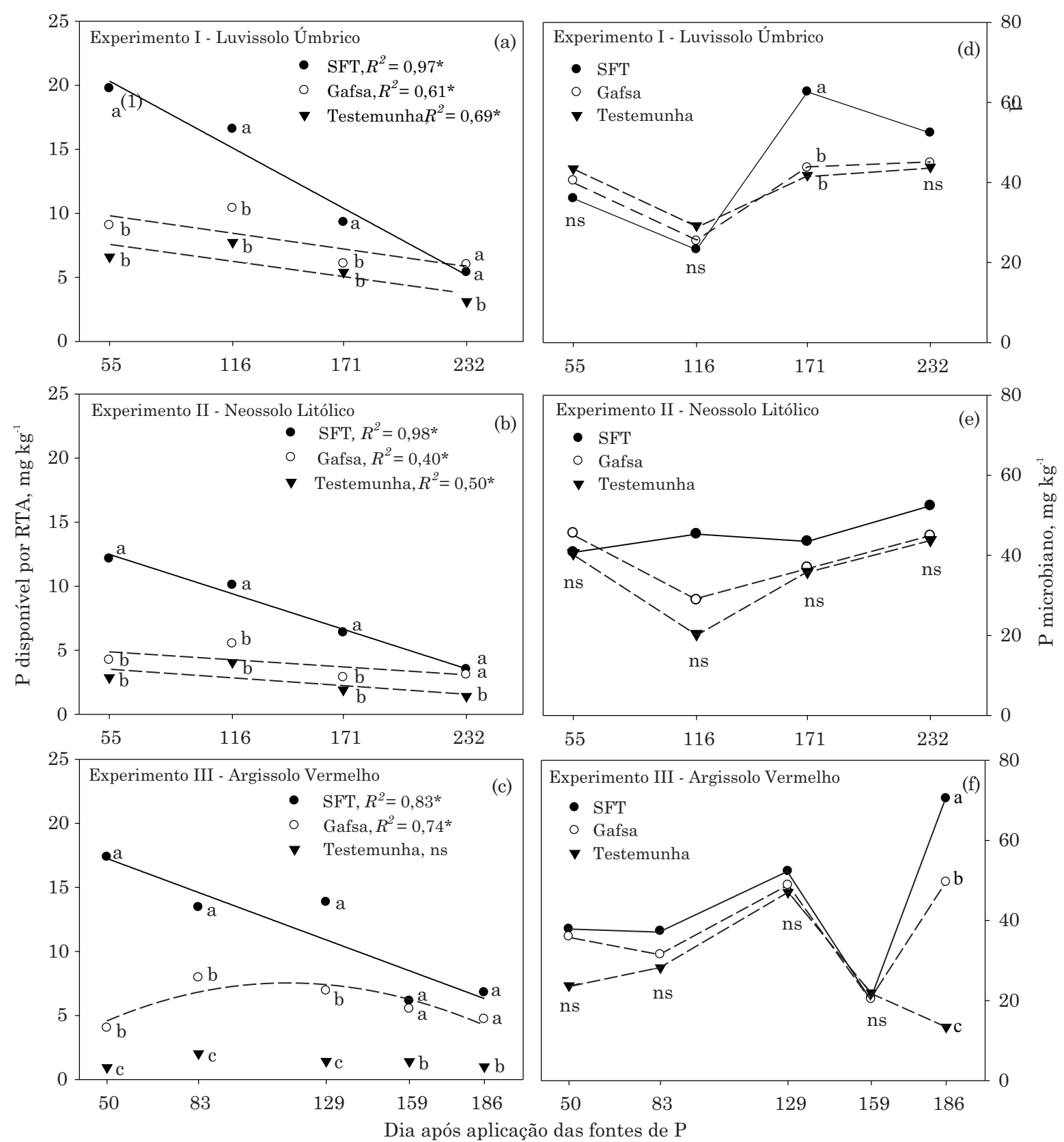

Figura 2. Variação do teor de $P$ extraído por RTA ( $a, b, c)$ e teor de $P$ armazenado na BMS (d, e, f), após a aplicação de fontes de fosfato em solos sob pastagem natural. Diferença significativa a $p<0,05$ entre épocas de avaliação. Médias seguidas pela mesma letra em cada época de avaliação, comparando as fontes de fosfato, não diferem significativamente entre si em nível de $\mathbf{p}<0,05$ pelo teste de Tukey; e ns: não significativo. 
Quadro 2. Significância dos efeitos dos fatores experimentais e sua interação sob as formas de $\mathrm{P}$ em solos sob pastagem natural como resultado da análise da variância

\begin{tabular}{|c|c|c|c|c|c|}
\hline Solo/Fonte de variação & P-RTA & $\mathbf{P}$ microbiano & $\mathrm{P}-\mathrm{NaOH}$ & P orgânico & P total \\
\hline \multicolumn{6}{|c|}{ Experimento I - Luvissolo Úmbrico } \\
\hline Fonte de fosfato & $* *$ & ns & $* *$ & $\mathrm{~ns}$ & ns \\
\hline Tempo & $* *$ & $* *$ & $\mathrm{~ns}$ & $* * *$ & $\mathrm{~ns}$ \\
\hline Fonte de fosfato $\mathrm{x}$ Tempo & $* *$ & $* *$ & ns & $\mathrm{ns}$ & ns \\
\hline \multicolumn{6}{|c|}{ Experimento II - Neossolo Litólico } \\
\hline Fonte de fosfato & $* * *$ & ns & * & $\mathrm{ns}$ & ns \\
\hline Tempo & $* *$ & $* *$ & ns & $* *$ & ns \\
\hline Fonte de fosfato $\mathrm{x}$ Tempo & $* *$ & ns & ns & $\mathrm{ns}$ & $\mathrm{ns}$ \\
\hline \multicolumn{6}{|c|}{ Experimento III - Argissolo Vermelho } \\
\hline Fonte de fosfato & $* * *$ & $* *$ & $* * *$ & $* *$ & $* *$ \\
\hline Tempo & $* *$ & ns & $\mathrm{ns}$ & $\mathrm{ns}$ & $\mathrm{ns}$ \\
\hline Fonte de fosfato $\mathrm{x}$ Tempo & $* *$ & $* *$ & ns & $\mathrm{ns}$ & $\mathrm{ns}$ \\
\hline
\end{tabular}

ns: não significativo. ${ }^{*},{ }^{* *}$ e ${ }^{* * *}$ : significativo a $\mathrm{p}<0,05, \mathrm{p}<0,01$ e $\mathrm{p}<0,001$, respectivamente.

Quadro 3. Teor de P extraído por $\mathrm{NaOH} \mathrm{0,1} \mathrm{mol} \mathrm{L-1,} \mathrm{P}$ orgânico total e $\mathbf{P}$ total em diferentes solos sob pastagem natural, submetidos à aplicação de diferentes fontes de fosfato

\begin{tabular}{lccc}
\hline Solo/Tratamento & $\begin{array}{c}\text { P-NaOH 0,1 } \\
\text { mol L }\end{array}$ & $\begin{array}{c}\mathbf{P} \\
\text { orgânico }\end{array}$ & $\begin{array}{c}\mathbf{P} \\
\text { total }\end{array}$ \\
\cline { 2 - 4 } & \multicolumn{3}{c}{$\mathrm{mg} \mathrm{kg}^{-1}$} \\
Experimento I - Luvissolo Úmbrico & & \\
Testemunha & $126 \mathrm{~b}$ & $120 \mathrm{a}$ & $330 \mathrm{a}$ \\
Gafsa & $117 \mathrm{~b}$ & $125 \mathrm{a}$ & $331 \mathrm{a}$ \\
SFT & $149 \mathrm{a}$ & $122 \mathrm{a}$ & $333 \mathrm{a}$ \\
Experimento II - Neossolo Litólico & & \\
Testemunha & $97 \mathrm{~b}$ & $130 \mathrm{a}$ & $273 \mathrm{a}$ \\
Gafsa & $102 \mathrm{~b}$ & $130 \mathrm{a}$ & $333 \mathrm{a}$ \\
SFT & $131 \mathrm{a}$ & $138 \mathrm{a}$ & $315 \mathrm{a}$ \\
Experimento III - Argissolo Vermelho & & \\
Testemunha & $60 \mathrm{c}$ & $81 \mathrm{c}$ & $225 \mathrm{~b}$ \\
Gafsa & $85 \mathrm{~b}$ & $89 \mathrm{~b}$ & $271 \mathrm{a}$ \\
SFT & $113 \mathrm{a}$ & $96 \mathrm{a}$ & $292 \mathrm{a}$ \\
\hline
\end{tabular}

Médias seguidas pela mesma letra na coluna em cada solo não diferem significativamente entre si a $\mathrm{p}<0,05$ pelo teste de Tukey.

O aumento do teor de $\mathrm{P}$ disponível com a aplicação de fosfatos solúveis em solos de ambientes de pastagens naturais com baixos teores de $\mathrm{P}$ pode aumentar a importância das frações inorgânicas lábeis do nutriente para a nutrição das plantas. Dessa forma, as espécies nativas, componentes das pastagens naturais, podem tornarem-se menos dependentes da mineralização das frações orgânicas de $\mathrm{P}$ para a produção de forragem (Tiecher et al., 2013).

O teor de P imobilizado pela BMS não acompanhou o aumento dos teores de $\mathrm{P}$ disponível na maioria dos casos, demonstrando-se um indicador pouco sensível à aplicação de diferentes fontes de fosfatos em pastagens naturais. Com exceção da última coleta de solo no experimento III em Santa Maria e da terceira coleta de solo no experimento I em Candiota, o teor de P imobilizado na BMS apresentou variações ao longo do tempo que ocorreram sem influência dos tratamentos. Isso ocorreu porque o aumento da imobilização de P na BMS depende de outros fatores além da disponibilidade de $\mathrm{P}$ nos solos, como as fontes de energia no sistema, ou seja, a oferta de $\mathrm{C}$ de fácil decomposição. Essa oferta possui estreita relação com os ciclos de crescimento e morte da vegetação, que por sua vez é consequência principalmente de fatores sob controle antrópico como roçadas (Rheinheimer et al., 2008), pastejo e queimadas (Oliveira et al., 2011) e de fatores abióticos como temperatura, radiação, disponibilidade de água, como no caso deste trabalho. Isso pode ser verificado pelo menor teor de $\mathrm{P}$ armazenado na BMS nas coletas de solo realizadas durante os períodos de temperaturas mais altas (aos 116 e 159 dias após a aplicação dos tratamentos nos experimentos de Candiota e Santa Maria, respectivamente - Figura 2 d,e,f) e pelas diferenças no teor de $\mathrm{P}$ armazenado pela BMS entre as fontes de fosfato aplicadas que só foram observadas nos períodos em que não havia déficit hídrico (aos 171 e 186 dias após a aplicação dos tratamentos no experimento I em Candiota e no experimento III em Santa Maria, respectivamente - Figura 2 d,f).

Como a BMS não foi dreno do $\mathrm{P}$ disponibilizado pela aplicação de fertilizantes fosfatados, a diminuição do teor de $\mathrm{P}$ disponível nos solos submetidos à aplicação de SFT provavelmente foi em razão da absorção do nutriente pelas plantas e da sua adsorção pelas partículas reativas do solo. No quadro 4, evidencia-se que a pastagem natural foi mais efetiva em utilizar o $\mathrm{P}$ disponibilizado pela aplicação de fosfato solúvel (SFT) a longo prazo no experimento III em Santa Maria, comparativamente ao histórico recente de aplicação de P nos experimentos I e II em Candiota. 
Quadro 4. Balanço de $\mathrm{P}$ em pastagem natural sob diferentes solos e submetidos à aplicação de diferentes fontes de fosfato

\begin{tabular}{|c|c|c|c|c|c|c|}
\hline \multirow{2}{*}{ Solo/Tratamento } & \multicolumn{3}{|c|}{$P$ disponível na camada $0-10 \mathrm{~cm}^{(1)}$} & \multirow{2}{*}{$\begin{array}{c}\text { MS da parte } \\
\text { aérea }^{(2)}\end{array}$} & \multirow{2}{*}{$\begin{array}{c}\text { Teor de } P \\
\text { na } \mathrm{MS}^{(2)}\end{array}$} & \multirow{2}{*}{$\begin{array}{c}\text { P acumulado } \\
\text { na MS }\end{array}$} \\
\hline & Primeira coleta & Última coleta & Variação & & & \\
\hline & 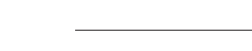 & - $\mathrm{kg} \mathrm{ha} \mathrm{a}^{-1}$ & - & $\mathrm{Mg} \mathrm{ha}^{-1}$ & $\%$ & $\mathrm{~kg} \mathrm{ha}^{-1}$ \\
\hline \multicolumn{7}{|c|}{ Experimento I - Luvissolo Úmbrico } \\
\hline Testemunha & 8,6 & 4,0 & $-4,6$ & 2,7 & 0,15 & 4,2 \\
\hline Gafsa & 11,8 & 7,8 & $-4,0$ & 2,6 & 0,14 & 3,5 \\
\hline SFT & 25,6 & 7,0 & $-18,6$ & 2,9 & 0,19 & 5,3 \\
\hline \multicolumn{7}{|c|}{ Experimento II - Neossolo Litólico } \\
\hline Testemunha & 3,8 & 1,8 & $-2,0$ & 2,2 & 0,11 & 2,3 \\
\hline Gafsa & 5,5 & 4,0 & $-1,4$ & 2,5 & 0,13 & 3,3 \\
\hline SFT & 15,7 & 4,6 & $-11,2$ & 2,6 & 0,12 & 3,3 \\
\hline \multicolumn{7}{|c|}{ Experimento III - Argissolo Vermelho } \\
\hline Testemunha & 1,2 & 1,3 & 0,1 & 2,9 & 0,09 & 2,5 \\
\hline Gafsa & 5,2 & 6,1 & 0,9 & 3,0 & 0,15 & 4,6 \\
\hline SFT & 22,6 & 8,8 & $-13,8$ & 3,9 & 0,19 & 7,2 \\
\hline
\end{tabular}

(1) Para o cálculo de $\mathrm{P}$ disponível no solo da camada de 0-10 cm foram utilizados o teor de $\mathrm{P}$ extraído por RTA e a densidade média de cada solo. ${ }^{(2)}$ Avaliações realizadas na data da última coleta de solo.

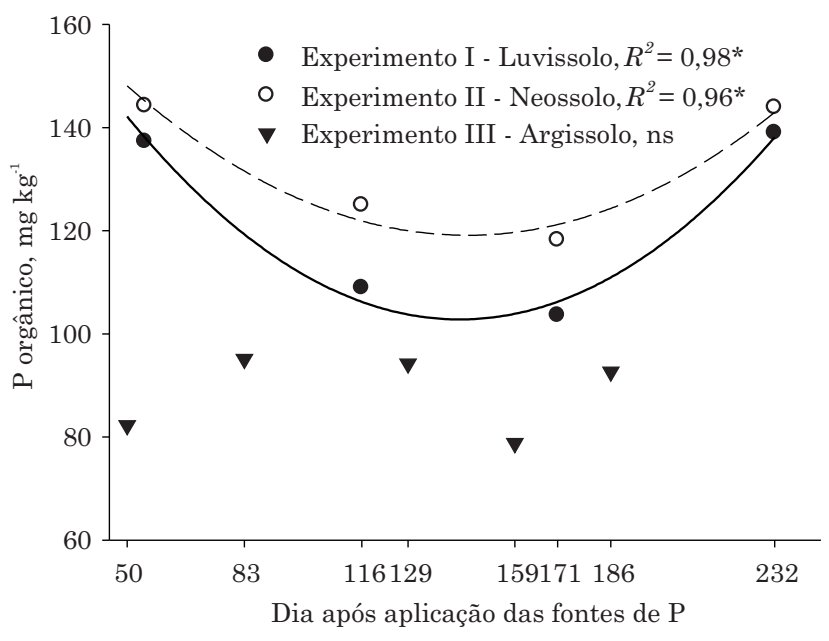

Figura 3. Variação do teor de $\mathbf{P}$ orgânico após a aplicação de fontes de fosfato em solos sob pastagem natural. Diferenças significativas a p<0,05 entre épocas de avaliação; e ns: não significativo.

Isso pode ser atribuído a dois principais motivos. Primeiramente, no experimento em Santa Maria, em razão do seu longo histórico de adubação fosfatada, parte dos sítios mais reativos do solo já foi saturada e, por isso, o $\mathrm{P}$ adicionado via SFT na última aplicação pode ter sido adsorvido com menor energia de ligação, apresentando maior disponibilidade para as plantas (Barrow et al., 1998). Tal fato é confirmado pelo incremento no teor de $\mathrm{P}$ moderadamente disponível estimado pela extração com $\mathrm{NaOH}$ quando da aplicação de SFT (Quadro 3), o que também foi observado por Gatiboni et al. (2007). Já nos dois experimentos em
Candiota, que não tinham sido fertilizados anteriormente, possivelmente existia grande quantidade de sítios de alta afinidade por fosfato, resultando em maior adsorção do $\mathrm{P}$ solúvel adicionado no solo. O outro possível motivo pode ter sido o menor crescimento da pastagem nos experimentos I e II em Candiota, diminuindo assim a eficiência da adubação fosfatada, pois o $\mathrm{P}$ solúvel que não foi absorvido pelas plantas ou pela BMS foi então adsorvido pelos coloides inorgânicos do solo.

O P extraído por $\mathrm{NaOH}$ é um bom indicativo da quantidade de $\mathrm{P}$ adsorvido pelos óxidos de $\mathrm{Fe}$ e $\mathrm{Al}$ do solo, e estima o teor de $\mathrm{P}$ moderadamente disponível no solo (Chang \& Jackson, 1957; Hedley et al., 1982b). Conforme Dias (1978), admitindo-se que todo o P solúvel adicionado ao solo na forma de SFT ou proveniente da dissolução de uma apatita de um fosfato natural seja adsorvido pelos óxidos de $\mathrm{Fe}$ e $\mathrm{Al}$, é possível acompanhar a dissolução de fosfatos de cálcio por meio da extração com $\mathrm{NaOH}$. Como nos experimentos de Candiota o teor de $\mathrm{P}$ extraído por $\mathrm{NaOH}$ com aplicação de FN não diferiu do tratamento testemunha (Quadro 3), verifica-se que a dissolução do FN nos experimentos I e II foi muito baixa. Somente no experimento de Santa Maria, com histórico de 13 anos de aplicação de $\mathrm{FN}$, o teor de $\mathrm{P}$ extraído por $\mathrm{NaOH}$ foi superior ao tratamento testemunha (Quadro 3). Esses resultados claramente evidenciam a lenta dissolução dos FN, mesmo em condições de solos ácidos e com baixos teores de $\mathrm{P}$ e $\mathrm{Ca}$.

Existem escassas informações a respeito dos fatores que favorecem o aumento do teor de $\mathrm{P}$ orgânico do solo, mas normalmente o incremento de $\mathrm{P}$ orgânico ocorre em sistemas onde os teores de C orgânico total e P total aumentam (Nziguheba \& Bünemann, 2005). 
Como nos dois experimentos conduzidos em Candiota a aplicação de $100 \mathrm{~kg} \mathrm{ha}^{-1}$ de $\mathrm{P}_{2} \mathrm{O}_{5}$ não aumentou o teor de $\mathrm{P}$ total nem a produção de biomassa, o teor de $\mathrm{P}$ orgânico total também não foi alterado pelos tratamentos. Já no experimento em Santa Maria, por causa do histórico de aplicação de $470 \mathrm{~kg} \mathrm{ha}^{-1} \mathrm{de}_{2} \mathrm{O}_{5}$, houve aumento de $25 \%$ do teor de P total com a aplicação de SFT e FN. Contudo, a aplicação de SFT incrementou de forma mais acentuada a produção de massa de forragem ao longo dos anos comparativamente aos FN (Gatiboni et al., 2000). Assim, em razão do maior aporte de resíduos senescentes das plantas ao solo, houve a transformação do P inorgânico aplicado em formas orgânicas com a aplicação de SFT, comparativamente à aplicação de FN (Quadro 3). Mesmo assim, apenas 17,4 e 22,4 \% do $\mathrm{P}$ adicionado via FN e SFT acumularam-se na forma orgânica, demonstrando que o $\mathrm{P}$ adicionado no solo está se armazenando principalmente em formas inorgânicas, como observado também por Rheinheimer \& Anghinoni (2001), Conte et al. (2003), Gatiboni et al. (2008), Ceretta et al. (2010) e Guardini et al. (2012).

Nos solos sob pastagens naturais exploradas pela atividade pecuária por longos períodos de tempo, o teor de $\mathrm{P}$ total do solo pode diminuir em razão do exaurimento das reservas de $\mathrm{P}$ orgânico e inorgânico pela absorção das plantas (Gatiboni et al., 2007;2008) e posterior exportação do elemento pela produção animal. Entretanto, durante o período avaliado não houve grande exportação da forragem produzida pelas pastagens, apenas a absorção do nutriente pelas plantas. De modo geral, o teor de P na massa seca da parte aérea da pastagem natural dos experimentos avaliados variou de 0,09 a $0,19 \%$, e o acúmulo de P na massa seca da parte área variou de 2,3 a 7,2 $\mathrm{kg} \mathrm{ha}^{-1}$, o que não foi suficiente para alterar o teor de $\mathrm{P}$ total do solo, mantendo-o constante em todas as épocas avaliadas e em todos os solos.

\section{CONCLUSÕES}

1. A aplicação de fosfato solúvel em água promoveu aumento da disponibilidade de P no solo sob pastagens naturais, inclusive mantendo níveis mais elevados em curto, médio e longo prazos, comparativamente ao fosfato natural.

2. A aplicação de fertilizantes fosfatados, principalmente os solúveis em água, em solos de baixa fertilidade natural, sob pastagens naturais, aumentou a importância das frações inorgânicas lábeis de $\mathrm{P}$ na disponibilização do nutriente para as plantas, tornando-as menos dependentes da mineralização das frações orgânicas.

3. O P microbiano não é bom indicador da biodisponibilidade de $\mathrm{P}$ em pastagens naturais sob Argissolos, Neossolos e Luvissolos do sul do Brasil, fertilizadas com fosfatos.

\section{LITERATURA CITADA}

BARROW, N.J.; BOLLAND, M.D.A. \& ALLEN, D.G. Effect of addition of superphosphate on sorption of phosphate. Aust. J. Soil Res., 36:359-372, 1998.

BROOKES, P.C.; POWLSON, D.S. \& JENKINSON, D.S. Measurement of microbial biomass phosphorus in soil. Soil Biol. Biochem., 14:319-329, 1982.

CERETTA, C.A.; LORENSINI, F.; BRUNETTO, G.; GIROTTO, E.; GATIBONI, L.C.; LOURENZI, C.R.; TIECHER, T.L.; DE CONTI, L.; TRENTIN, G. \& MIOTTO, A. Frações de fósforo no solo após sucessivas aplicações de dejetos de suínos em plantio direto. Pesq. Agropec. Bras., 45:593$602,2010$.

CHANG, S.C. \& JACKSON, M.L. Fractionation of soil phosphorus. Soil Sci., 84:133-144, 1957.

CHIEN, S.H.; PROCHNOW, L.I.; TU, S. \& SNYDER, C.S. Agronomic and environmental aspects of phosphate fertilizers varying in source and solubility: an update review. Nutr. Cycl. Agroecosyst., 89:229-255, 2011.

COMISSÃO DE QUÍMICA E FERTILIDADE DO SOLO CQFSRS/SC. Manual de adubação e de calagem para os Estados do Rio Grande do Sul e Santa Catarina. Porto Alegre, SBCS/NRS, 2004. 400p.

CONTE, E.; ANGHINONI, I. \& RHEINHEIMER, D.S. Fósforo da biomassa microbiana e atividade de fosfatase ácida após aplicações de fosfato em solo no sistema plantio direto. R. Bras. Ci. Solo, 26:925-930, 2002.

CONTE, E.; ANGHINONI, I. \& RHEINHEIMER D.S. Frações de fósforo acumuladas em Latossolo argiloso pela aplicação de fosfato no sistema plantio direto. R. Bras. Ci. Solo, 27:893-900, 2003.

DIAS, V.M.C. Solubilidade de fosfatos naturais apatíticos em acetato de amônia em solos. Santa Maria, Universidade Federal do Rio Grande do Sul, 1978. 56p. (Dissertação de Mestrado)

GATIBONI, L.C.; KAMINSKI, J.; RHEINHEIMER, D.S. \& FLORES, J.P.C. Biodisponibilidade de formas de fósforo acumuladas em solo sob sistema plantio direto. R. Bras. Ci. Solo, 31:691-699, 2007.

GATIBONI, L.C.; KAMINSKI, J.; RHEINHEIMER, D.S. \& BRUNETTO, G. Fósforo da biomassa microbiana e atividade de fosfatase ácidas durante a diminuição do fósforo disponível no solo. Pesq. Agropec. Bras., 43:10851091, 2008.

GATIBONI, L.C.; KAMINSKI, J.; PELLEGRINI, J.B.R.; BRUNETTO, G.; SAGGIN, A. \& FLORES, J.P.C. Influência da adubação fosfatada e da introdução de espécies forrageiras de inverno na oferta de forragem de pastagem natural. Pesq. Agropec. Bras., 35:1663-1668, 2000 .

GATIBONI, L.C.; KAMINSKI, J.; RHEINHEIMER, D.S. \& BRUNETTO, G. Superphosphate and rock phosphates as P-source for grass-clover pasture on a limed acid soil of Southern Brazil. Commun. Soil Sci. Plant Anal., 42:1$12,2003$. 
GUARDINI, R.; COMIN, J.J.; SCHMITT, D.E.; TIECHER, T.; BENDER, M.A.; RHEINHEIMER, D.S.; MEZZARI, C.P.; OLIVEIRA, B.S.; GATIBONI, L.C. \& BRUNETTO, G. Accumulation of phosphorus fractions in typic Hapludalf soil after long-term application of pig slurry and deep pig litter in a no-tillage system. Nutr. Cycl. Agroecosyst., 93:215-225, 2012.

HEDLEY, M.J. \& STEWART, J.W.B. Method to measure microbial phosphate in soils. Soil Biol. Biochem., 14:377$385,1982 \mathrm{a}$.

HEDLEY, M.J.; STEWART, J.W.B. \& CHAUHAN, B.S. Changes in inorganic and organic soil phosphorus fractions induced by cultivation practices and by laboratory incubations. Soil Sci. Soc. Am. J., 46:970-976, 1982b.

JONES, D.L. Organic acids in the rhizosphere - a critical review. Plant Soil, 205:25-44, 1998.

KUO, S.; HUANG, B. \& BEMBENEK, R. Effects of long-term phosphorus fertilization and winter cover cropping on soil phosphorus transformations in less weathered soil. Biol. Fertil. Soils, 4:116-123, 2005.

MURPHY, J. \& RILEY, J.P. A modified single solution method for the determination of phosphate in natural waters. Anal. Chim. Acta, 27:31-36, 1962.

MARTINAZZO, R.; RHEINHEIMER, D.S.; GATIBONI, L.; BRUNETO, G. \& KAMINSKI, J. Fósforo microbiano do solo sob sistema plantio direto em resposta à adição de fosfato solúvel. R. Bras. Ci. Solo, 31:563-570, 2007.

MOREL, C.; TIESSEN, H. \& STEWART, J.W.B. Correction for $\mathrm{P}$ sorption in the measurement of soil microbial biomass $\mathrm{P}$ by $\mathrm{CHCl}_{3}$ fumigation. Soil Biol. Biochem. 28:1699-1706, 1996.

NZIGUHEBA, G. \& BÜNEMANN, E.K. Organic phosphorus dynamics in tropical agroecosystems. In: TURNER, B.L.; FROSSARD, E. \& BALDWIN, D.S., eds. Organic phosphorus in the environmental. Wallingford, CAB International, 2005. p.243-268.

OLIVEIRA, L.B.; TIECHER, T.; QUADROS, F.L.F. \& RHEINHEIMER, D.S. Fósforo microbiano em solos sob pastagem natural afetado por queima e pastejo. R. Bras. Ci. Solo, 35:1509-1515, 2011.

OLSEN, S.R. \& SOMMERS, L.E. Phosphorus. In: PAGE, A.L.; MILLER, R.H. \& KEENEY, Q.R., eds. Methods of soil analysis. Chemical and microbiological properties. Madison, Soil Science Society of America, 1982. Part 2. p.403-430.

PELLEGRINI, L.G.; NABINGER, C.; NEUMANN, M.; CARVALHO, P.C.F. \& CRANCIO, L.A. Produção de forragem e dinâmica de uma pastagem natural submetida a diferentes métodos de controle de espécies indesejáveis e à adubação. R. Bras. Zootec., 39:2380-2388, 2010.

RHEINHEIMER, D.S.; ANGHINONI, I. \& KAMINSKI, J. Depleção de fósforo inorgânico de diferentes frações provocada pela extração sucessiva com resinas em diferentes solos e manejos. R. Bras. Ci. Solo, 24:345-354, 2000.

RHEINHEIMER, D.S. \& ANGHINONI, I. Distribuição do fósforo inorgânico em sistemas de manejo de solo. Pesq. Agropec. Bras., 36:151-160, 2001.
RHEINHEIMER, D.S.; ANGHINONI, I.; CONTE, E.; KAMINSKI, J. \& GATIBONI, L.C. Dessorção de fósforo avaliada por extrações sucessivas em amostras de solo provenientes dos sistemas plantio direto e convencional. Ci. Rural, 33:1053-1059, 2003a.

RHEINHEIMER, D.S.; ANGHINONI, I. \& CONTE, E. Sorção de fósforo em função do teor inicial e de sistemas de manejo de solos. R. Bras. Ci. Solo, 27:41-49, 2003b.

RHEINHEIMER, D.S.; MARTINAZZO, R.; GATIBONI, L.C.; KAMINSKI, J. \& SILVA, L.S. Amplitude no fósforo microbiano em um Argissolo em pastagem nativa submetida à roçada e à introdução de espécies forrageiras com fertilização fosfatada em diferentes épocas. Acta Sci. Agron., 30:561-567, 2008.

SOARES, A.B.; CARVALHO, P.C.F.; NABINGER, C.; SEMMELMANN, C.; TRINDADE, J.K.; GUERRA, E.; FREITAS, T.S.; PINTO, C.E.; FONTOURA JÚNIOR, J.A. \& FRIZZO, A. Produção animal e de forragem em pastagem nativa submetida a distintas ofertas de forragem. Ci. Rural, 35:1148-1154, 2005.

SORN-SRIVICHAI, P.; SYERS, J.K.; TILMAN, R.W. \& CORNFORTH, I.S. An evaluation of water extraction as a soil testing procedure for phosphorus. II - Factors affecting the amounts of water-extractable phosphorus in fields soils. Fert. Res., 15:225-236, 1988.

SZILAS, C.; SEMOKA, J.M.R. \& BORGGAARD, O.K. Can local Minjingu phosphate rock replace superphosphate on acid soils in Tanzania? Nutr. Cycl. Agroecosyst., $77: 257-268,2007$

TAKEDA, M.; NAKAMOTO, T.; MIYAZAWA, K. \& MURAYAMA, T. Phosphorus transformation in a soybean-cropping system in Andosol: Effects of winter cover cropping and compost application. Nutr. Cycl. Agroecosyst., 85:287-297, 2009.

TEDESCO, M.J.; GIANELLO, C.; BISSANI, C.A.; BOHNEN, H. \& VOLKWEISS, S.J. Análise de solo, plantas e outros materiais. Porto Alegre, Universidade Federal do Rio Grande do Sul, 1995. 174p.

TIECHER, T.; RHEINHEIMER, D.S.; KAMINSKI, J. \& CALEGARI, A. Forms of inorganic phosphorus in soil under different long term soil tillage systems and winter crops. R. Bras. Ci. Solo, 36:271-281, 2012a.

TIECHER, T.; RHEINHEIMER, D.S. \& CALEGARI, A. Soil organic phosphorus forms under different soil management systems and winter crops, in a long term experiment. Soil Till. Res., 124:57-67, 2012b.

TIECHER, T.; OLIVEIRA, L.B.; RHEINHEIMER, D.S.; QUADROS, F.L.F.; GATIBONI, L.C.; BRUNETTO, G. \& KAMINSKI, J. Phosphorus application and liming effects on forage production, floristic composition and on soil chemical properties in Campos Biome, Southern Brazil. Grass. For. Sci., doi: 10.1111/gfs.12079, 2013.

UNITED STATES ENVIRONMENTAL PROTECTION AGENCY - USEPA. Methods of chemical analysis for water and wastes. Cincinnati, 1971. 
VU, D.T.; TANG, C. \& ARMSTRONG, R.D. Transformations and availability of phosphorus in three contrasting soil types from native and farming systems: A study using fractionation and isotopic labeling techniques. J. Soils Sed., 10:18-29, 2010.
WANG, J.; LIU, W.-Z.; MU, H.-F. \& DANG T.-H. Inorganic phosphorus fractions and phosphorus availability in a calcareous soil receiving 21-years superphosphate application. Pedosphere, 20:304-310, 2010. 